\title{
Resultados perioperatorios y sobrevida alejada de la pancreatoduodenectomía con resección vascular*
}

\author{
Drs. ENRIQUE NORERO M. ${ }^{1}$, SERGIO BÁEZ V. ${ }^{1}$, EDUARDO VIÑUELA F. ${ }^{1}$, CRISTIAN MARTÍNEZ B. ${ }^{1}$, \\ JULIO REYES R. ${ }^{1}$, RODRIGO KUSANOVIC B. ${ }^{1}$, MARCEL SANHUEZA G. ${ }^{1}$, GLORIA AGUAYO B. ${ }^{2}$, \\ ALFONSO CALVO B. ${ }^{1}$, ROSE MARIE MEGE R. ${ }^{1}$, MARIO CARACCI L. ${ }^{1}$, ALFONSO DÍAZ F. ${ }^{1}$
}

1 Servicio de Cirugía.

2 Servicio de Anatomía Patológica.

Hospital Sótero del Río. Santiago, Chile.

\begin{abstract}
Pancreaticoduodenectomy with vascular resection. Perioperative results and longterm survival
\end{abstract}

Background: Pancreaticoduodenectomy (PDD) with vascular resection (VR) of the portal and superior mesenteric vein for locally advanced periampullary tumors is controversial. Aim: To evaluate the perioperative results and long-term survival of PDD with VR. Patients and Methods: Retrospective study. We included patients with periampullary tumors who underwent a PDD with VR between 1990 and 2008. We compared perioperative results and long-term survival with PDD without VR during the same period. We compared survival with non resected patients. Results: One hundred and eighty eight patients underwent a PDD, a VR was performed in 8 (4\%) patients (Age: $58 \pm 14$ years, Male: 4). Morbidity for PDD with and without VR was $75 \%$ and $59 \%(\mathrm{p}=\mathrm{ns})$. Surgical mortality for PDD with and without VR was $0 \%$ and $8 \%(\mathrm{p}=\mathrm{ns})$. In 6 of 8 patients the diagnosis was pancreatic cancer and histopathologic confirmation of vascular invasion was present in 4 patients. Long-term survival for patients with PDD with and without VR was similar (median 25 and 16 months; $\mathrm{p}=\mathrm{ns}$ ). Survival for patients with PDD with VR was superior to non resected patients (median 25 and 3 months; $p=0.0001$ ). Conclusions: PDD with VR has similar perioperative results and long-term survival to PDD without VR. The survival reached with this type of surgery is far superior to non resected patients.

Key words: Periampullary tumor, pancreatic cancer, pancreaticoduodenectomy, Whipple, portal vein, superior mesenteric vein.

\section{Resumen}

Introducción: La pancreatoduodenectomía (PDD) con resección vascular (RV) cuando existe invasión de la vena porta o mesentérica superior (VP-VMS) es controversial. Objetivo: Evaluar los resultados del perioperatorio y la sobrevida alejada de los pacientes sometidos a esta técnica. Material y Método: Estudio retrospectivo que incluyó a los pacientes con un tumor periampular en quienes se realizó una PDD con RV entre 1990 y 2008. Se compararon los resultados del perioperatorio y de sobrevida alejada con el

*Recibido El 9 de Junio de 2009 y aceptado para publicación el 4 de Agosto de 2009.

Correspondencia: Dr. Enrique Norero M.

Av. Concha y Toro 3459, Puente Alto, Santiago, Chile. Fax: 5622897995

E-mail: enorero@yahoo.com 
grupo sometido a una PDD sin RV durante el mismo período. Se comparó también la sobrevida con los pacientes no resecados. Resultados: Se realizaron 188 PDD, en 8 (4\%) de estos pacientes se realizó PDD con RV (Edad: $58 \pm 14$ años, Hombres: 4). La morbilidad postoperatoria para la PDD con y sin RV fue de $75 \%$ y $59 \%(p=n s)$. La mortalidad postoperatoria para los grupos con y $\sin R V$ fue de $0 \%$ y $8 \%(p=n s)$. En 6 de los 8 pacientes el diagnóstico fue cáncer de páncreas y en 4 se confirmó histopatológicamente la invasión de VP-VMS. La sobrevida del grupo con y sin RV no tuvo diferencia significativa (medianas 25 y 16 meses; $\mathrm{p}=\mathrm{ns}$ ). La sobrevida de los pacientes sometidos a una PDD con RV fue superior a los pacientes no resecados (medianas 25 y 3 meses; $p=0,0001$ ). Conclusiones: La PDD con RV obtiene resultados perioperatorios y de sobrevida alejada comparables a una PDD sin RV. La sobrevida alcanzada con esta técnica es ampliamente superior a la de los pacientes no resecados.

Palabras clave: Neoplasia periampular, cáncer de páncreas, pancreatoduodenectomía, Whipple, vena porta, vena mesentérica superior.

\section{Introducción}

Los resultados perioperatorios de la pancreatoduodenectomía (PDD) para el tratamiento de los tumores periampulares han mejorado en forma notable durante los últimos años, especialmente en los centros de alto volumen de cirugía pancreática ${ }^{1}$.

Un especial desafío quirúrgico son aquellos pacientes que presentan invasión tumoral de la vena porta o de la vena mesentérica superior (VP-VMS). Clásicamente esta condición ha sido considerada un motivo de irresecabilidad. Sin embargo, existen reportes que apoyan que la PDD acompañada de una resección vascular (RV), con el objetivo de realizar una cirugía R0, no aumenta la morbimortalidad quirúrgica y permite alcanzar una sobrevida comparable a los pacientes que no presentan invasión de VP-VMS ${ }^{2-6}$.

El objetivo de este trabajo es evaluar los resultados del perioperatorio y la sobrevida alejada de los pacientes sometidos PDD con RV.

\section{Material y Método}

Estudio retrospectivo que incluyó a los pacientes con un tumor periampular en quienes se realizó una PDD con RV de VP-VMS entre enero 1990 y junio 2008, debido a sospecha de invasión tumoral de estos vasos. Se obtuvieron los datos desde la base de datos prospectiva de tumores periampulares de la oficina del cáncer de nuestro centro.

Se analizaron datos del paciente, características de la cirugía, morbilidad y mortalidad, duración de la hospitalización, evaluación de anatomía patológica (diagnóstico de tipo de tumor periampular, compromiso de adenopatías, estado del margen, invasión de VP-VMS) y sobrevida alejada. Se utilizó la clasificación de TNM sexta edición.

Se consideró mortalidad operatoria a la acontecida durante la hospitalización o hasta 1 mes después de la intervención quirúrgica.
El seguimiento se realizó mediante los registros clínicos, información del médico tratante y los certificados de defunción del Registro Civil. Se calculó la sobrevida con el seguimiento hasta julio de 2008 y se incluyó la mortalidad operatoria en este cálculo.

Se compararon los resultados del perioperatorio y de sobrevida alejada con los pacientes sometidos a una PDD sin RV durante el mismo período ( $\mathrm{n}$ $=180$ ). Para el análisis de sobrevida se excluyó a los pacientes con un diagnóstico final de patología benigna, tanto del grupo sin RV $(\mathrm{n}=12)$, como el grupo con $\mathrm{RV}(\mathrm{n}=1)$. Se comparó la sobrevida con un grupo de 156 pacientes no resecados que fueron evaluados durante el mismo período. En 17 de ellos se pudo precisar que el motivo de irresecabilidad fue exclusivamente invasión local.

\section{Técnica quirúrgica:}

La técnica de PDD utilizada ha sido descrita previamente $^{7}$, se realiza preservación pilórica de acuerdo a la preferencia de cada cirujano y desde algunos años atrás se utiliza una anastomosis pancreatogástrica $^{8}$. En el caso de sospecha de invasión vascular se completa la parte resectiva de la PDD y se realiza un control vascular proximal y distal al sitio del compromiso tumoral. Dependiendo de la magnitud del compromiso de los vasos se decide entre una resección lateral de la porta o una resección completa con una anastomosis termino-terminal. No ha sido necesario hasta ahora utilizar ninguna prótesis vascular. Es importante la completa liberación de la vena porta del resto del pedículo hepático y una disección amplia de la vena mesentérica superior, con el objetivo de realizar una venorrafia o una anastomosis vascular sin tensión. Se utiliza material Prolene ${ }^{\circledR}$ 5.0 ó 6.0. La PDD con $\mathrm{RV}$ se reserva para pacientes sin patología grave asociada y que están en buenas condiciones, en quienes no existe compromiso de la arteria mesentérica superior, y en los que se puede lograr una cirugía R0 resecando la VP-VMS. Se utilizó profi- 
laxis de enfermedad tromboembólica a dosis estándar de heparina en el postoperatorio.

\section{Análisis estadístico:}

Se utilizó los programas estadísticos Statview 5.0.1 y Minitab 15. En la comparación de datos se aplicaron las pruebas de $\chi^{2}$, t-Student y Fisher según correspondiera. Se calculó la sobrevida alejada con el método de Kaplan-Meier y se compararon con el test de log-rank. Se consideró significativo un valor $p \leq 0,05$. La sobrevida se expresa en medianas.

\section{Resultados}

Durante el período del estudio se realizaron 188 PDD (Mujeres: 101; Edad: $58 \pm 12$ años). En 8 (4\%) de estos pacientes se llevó a cabo una PDD con RV (Mujeres 4; Edad $58 \pm 14$ ) (Tablas 1 y 2).

Se utilizó una técnica de resección lateral de la porta en 7 de los 8 casos y en un paciente se resecó en forma circunferencial $2,5 \mathrm{~cm}$ de vena porta y se realizó una anastomosis termino-terminal. En este último caso se realizó también una resección parcial de vena cava inferior (Tabla 1).

Los pacientes con PDD con RV presentaron morbilidad en 6 de los 8 casos (75\%). En el intraoperatorio no existió sangrado mayor. En un caso se produjo una trombosis parcial en la zona de sutura lateral de la porta, que se trató mediante un catéter de Fogarty extrayendo el trombo y rehaciendo la sutura durante la misma cirugía. Dos pacientes presentaron retención gástrica, un paciente evolucionó con una fístula pancreática y un paciente presentó un hemoperitoneo no relacionado a la resec- ción de VP-VMS. Un paciente presentó una trombosis en la zona de sutura de la vena cava inferior que se trató con anticoagulación (Tabla 1). La morbilidad de los pacientes sometidos a una PDD sin RV fue de $59 \%$, no hubo diferencia estadísticamente significativa con el grupo de PDD con $\mathrm{RV}(\mathrm{p}=\mathrm{ns})$ (Tabla 2).

No existió mortalidad postoperatoria en el grupo de PDD con RV. En el grupo de PDD sin RV la mortalidad fue de $8 \%$, no hubo diferencia estadísticamente significativa $(\mathrm{p}=\mathrm{ns})$ (Tabla 2).

El promedio de estadía hospitalaria fue de $33 \pm$ 25 días en los pacientes con resección de VP-VMS, no hubo diferencia estadísticamente significativa con el grupo $\sin \mathrm{RV}(\mathrm{p}=\mathrm{ns})($ Tablas 1 y 2$)$.

El análisis de las piezas operatorias se muestra en la Tabla 3. En 6 de los 8 casos el diagnóstico fue cáncer de cabeza de páncreas, en su mayoría con ganglios positivos. Se lograron márgenes negativos en todos los casos. En 4 pacientes se confirmó la infiltración tumoral de la VP-VMS.

El seguimiento fue del $100 \%$. La sobrevida global de los pacientes sometidos a una PDD fue de 16 meses (23\% a 5 años). En los pacientes con una PDD con RV la mediana de sobrevida fue 25 meses (34\% a 5 años). Hay 3 pacientes que tuvieron una sobrevida superior a 2 años $(37 \%)$ y 2 con sobrevida sobre 5 años (25\%). Para los pacientes con una PDD sin RV la sobrevida fue de 16 meses ( $23 \%$ a 5 años), sin existir diferencia estadísticamente significativa $(\mathrm{p}=\mathrm{ns})$ (Figura 1). Al comparar la sobrevida con los pacientes no resecados, estos últimos presentaron una mediana de sobrevida de 3 meses, significativamente peor que los pacientes sometidos a una PDD con RV ( $\mathrm{p}=0,0001)$ (Figura 2), esto también fue así al comparar la sobrevida con los

Tabla 1. Resultados del perioperatorio de pacientes sometidos a pancreatoduodenectomía con resección vascular

\begin{tabular}{cccccc}
\hline Caso & Edad/Sexo & Resección vascular & Morbilidad & Mortalidad & Hospitalización \\
\hline 1 & $69 / \mathrm{M}$ & Lateral & $\mathrm{Si}^{*}$ & No & $18 \mathrm{~d}$ \\
2 & $53 / \mathrm{F}$ & Lateral & No & No & $28 \mathrm{~d}$ \\
3 & $70 / \mathrm{F}$ & Lateral & $\mathrm{Si}^{*}$ & No & $28 \mathrm{~d}$ \\
4 & $67 / \mathrm{F}$ & Lateral & No & No & $10 \mathrm{~d}$ \\
5 & $69 / \mathrm{M}$ & Lateral & $\mathrm{Si}^{*}$ & No & $75 \mathrm{~d}$ \\
6 & $59 / \mathrm{F}$ & Lateral & $\mathrm{Si}^{*}$ & No & $38 \mathrm{~d}$ \\
7 & $49 / \mathrm{M}$ & Lateral & $\mathrm{Si}^{*}$ & No & $20 \mathrm{~d}$ \\
8 & $28 / \mathrm{M}$ & Completa & $\mathrm{Si}^{*}$ & No & $57 \mathrm{~d}$ \\
\hline
\end{tabular}

M: Masculino. F: Femenino. *Morbilidad: paciente 1 (Hemoperitoneo), paciente 3 (Falla respiratoria en VM por 48 hrs), paciente 5 y 6 (Retención gástrica), paciente 7 (Trombosis portal intraoperatoria, trombectomía) paciente 8 (Trombosis VCI, fistula pancreática, colección intrabdominal, HDA). \$Resección asociada de vena cava inferior. 
Tabla 2. Comparación de los pacientes sometidos a una pancreatoduodenectomía con y sin resección vascular

\begin{tabular}{|c|c|c|c|}
\hline & PDD con RV & PDD sin RV & $\mathbf{p}$ \\
\hline Pacientes & 8 & 180 & \\
\hline Sexo femenino & $4(50 \%)$ & $97(54 \%)$ & ns \\
\hline Edad (años) & $58 \pm 14$ & $58 \pm 12$ & ns \\
\hline Preservación pilórica & $(57 \%)$ & $(61 \%)$ & ns \\
\hline Anastomosis $\mathrm{P}-\mathrm{G}$ & $6(75 \%)$ & $162(90 \%)$ & ns \\
\hline Morbilidad & $6(75 \%)$ & $107(59 \%)$ & ns \\
\hline Fístula pancreática & $1(13 \%)$ & $24(13 \%)$ & ns \\
\hline Retención gástrica & $2(25 \%)$ & $48(27 \%)$ & ns \\
\hline Hemoperitoneo & $1(13 \%)$ & $8 \quad(4 \%)$ & ns \\
\hline Hemorragia digestiva & $1(13 \%)$ & $16 \quad(9 \%)$ & ns \\
\hline Colecciones & $1(13 \%)$ & $20(11 \%)$ & ns \\
\hline Mortalidad & $0 \quad(0 \%)$ & $15 \quad(8 \%)$ & ns \\
\hline Hospitalización (días) & $33 \pm 25$ & $27 \pm 20$ & ns \\
\hline \multicolumn{4}{|l|}{ Anatomía patológica } \\
\hline Cáncer de páncreas & $6(66 \%)$ & $70(39 \%)$ & ns \\
\hline Cáncer de ampolla & $0 \quad(0 \%)$ & $50(28 \%)$ & ns \\
\hline Cáncer de vía biliar & $0 \quad(0 \%)$ & $35(12 \%)$ & ns \\
\hline Patología benigna & $1(11 \%)$ & $12(6 \%)$ & ns \\
\hline Otros & $1(11 \%)$ & $13(6 \%)$ & ns \\
\hline Ganglios positivos & $(71 \%)$ & $(49 \%)$ & ns \\
\hline Bordes positivos & $0 \quad(0 \%)$ & $20(12 \%)$ & ns \\
\hline
\end{tabular}

P-G: Pancreato gástrica. PDD: Pancreatoduodenectomía. RV: Resección vascular. Resultados en promedio \pm desviación estándar y n $(\%)$.

Tabla 3. Detalles de anatomía patológica y seguimiento alejado de pacientes sometidos a pancreatoduodenectomía con resección vascular

\begin{tabular}{cllll}
\hline Caso & Diagnóstico & Bordes & Invasión vascular* & Seguimiento (m) \\
\hline 1 & Cáncer páncreas (T3N0) & Negativo & Con tumor & Muerto $67 \mathrm{~m} \dagger$ \\
2 & Cistoadenocarcinoma (T3N0) & Negativo & Sin tumor & Vivo $158 \mathrm{~m}$ \\
3 & Cáncer páncreas (T3N1) & Negativo & Con tumor & Muerto $8 \mathrm{~m}$ \\
4 & Cáncer páncreas (T3N1) & Negativo & Sin tumor & Muerto $9 \mathrm{~m}$ \\
5 & Cáncer páncreas (T3N1) & Negativo & Con tumor & Muerto $25 \mathrm{~m}$ \\
6 & Cáncer páncreas (T3N1) & Negativo & Sin tumor & Muerto $13 \mathrm{~m}$ \\
7 & Pancreatitis crónica & No aplica & No aplica & Vivo $14 \mathrm{~m}$ \\
8 & Cáncer páncreas (T3N1) & Negativo & Con tumorł & Vivo $9 \mathrm{~m}$ \\
\hline
\end{tabular}

*Según evaluación de anatomía patológica. †Muerte no relacionada (infarto agudo miocardio). $¥$ Resección asociada de vena cava inferior, invasión tumoral de la porta y vena cava. 


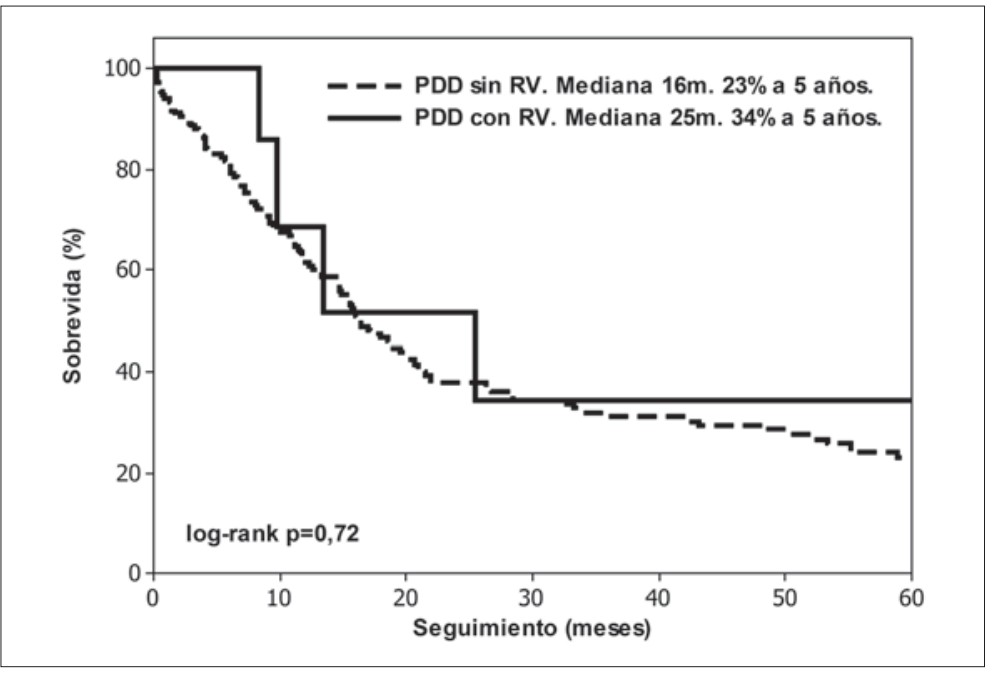

Figura 1. Sobrevida de pacientes sometidos a pancreatoduodenectomía con y sin resección vascular. PDD: pancreatoduodenectomía. RV: resección vascular.

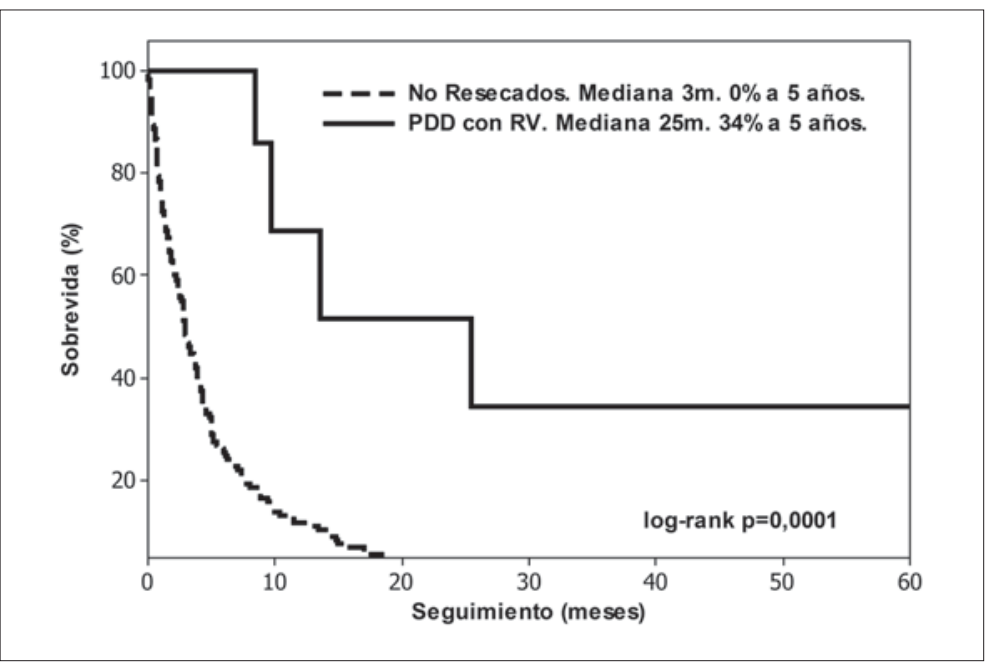

Figura 2. Sobrevida de pacientes sometidos a pancreatoduodenectomía con resección vascular y pacientes con tumores periampulares no resecados. PDD: pancreatoduodenectomía. RV: resección vascular. pacientes no resecados debido a compromiso local sin diseminación a distancia que presentaron una mediana de sobrevida de 4 meses $(p=0,002)$.

\section{Discusión}

Debido a su estrecha relación anatómica es frecuente que los tumores periampulares presenten invasión de la VP-VMS y de la arteria mesentérica superior (AMS). En el preoperatorio las imágenes de tomografía axial computada permiten una buena evaluación de esta zona ${ }^{9}$. La evaluación intraoperatoria de la invasión de estos vasos es clave para resolver casos dudosos ${ }^{10}$. Al existir invasión de la
AMS en la mayoría de los centros, incluyendo el nuestro, es considerado irresecable ${ }^{2,4}$ debido a que la resección de la AMS es al menos altamente controversial $^{11}$. La existencia de invasión aislada de la VP-VMS, con una confluencia esplenoportal permeable no representa una contraindicación absoluta a la resección pancreática, y su resección representa una manera apropiada de aumentar la resecabilidad y asegurarse márgenes negativos. Considerando que la invasión de la VP-VMS se produce debido a la ubicación del tumor y no necesariamente refleja su agresividad ${ }^{4,10}$.

Existe una importante variación en los distintos centros del porcentaje de pacientes en quienes se realiza una resección vascular de VP-VMS en pa- 
cientes sometidos a una PDD debido a tumores localmente avanzados. Esto refleja lo controversial de esta técnica. En esta serie representa el 4\% del total de las PDD realizadas, lo que está dentro de la amplia cifra descrita entre 3 y $38 \% \%^{2,11,12}$.

La morbilidad y mortalidad, descrita por una revisión sistemática sobre PDD con RV fue de $42 \%$ (rango: 9-78\%) y de 6\% respectivamente ${ }^{13}$. Los datos reportados en este trabajo son comparables a estos. Existe una importante variación según el centro, dada en gran parte por el volumen de cirugía pancreática ${ }^{14,15}$. En este trabajo no encontramos una diferencia significativa en la mortalidad ni en la morbilidad de la cirugía al comparar PDD con y sin RV.

También existe una amplia variación en la confirmación histopatológica de la infiltración vascular, describiéndose cifras entre $26-85 \%^{2-4,11,12,16-18}$. En esta serie el patólogo confirmó la invasión vascular en 4 pacientes. Debido a la dificultad de diferenciar entre invasión tumoral e inflamación peritumoral en el intraoperatorio se recomienda la resección de VPVMS cuando se sospeche macroscópicamente ${ }^{2}$.

Según nuestros datos confirmamos que la sobrevida a largo plazo de estos pacientes es comparable a aquellos en los que se realiza una PDD sin RV. La mediana de 25 y 16 meses de sobrevida, respectivamente, que nosotros reportamos es comparable a 13 meses de mediana descrita por grandes centros ${ }^{3,13,18}$. Por otro lado, la sobrevida con PDD con RV fue muy superior a la de los pacientes no resecados, tal como ha sido reportado por otros autores ${ }^{2-4,12,16}$. Si bien existen limitaciones al comparar ambos grupos, debido a que el grupo control ideal serían pacientes no resecados por compromiso exclusivo de VP-VMS, esto es muy difícil de identificar en un estudio de carácter retrospectivo. A pesar de esto, es conocido el mal pronóstico que tienen los pacientes no resecados, con medianas que no superan el año de sobrevida ${ }^{19}$.

Un caso especial son los pacientes con tumores quísticos y endocrinos del páncreas, de conocido mejor pronóstico que el adenocarcinoma de páncreas $^{20-22}$, estos pacientes representan buenos candidatos a una PDD con $\mathrm{RV}^{23}$; en esta serie el paciente con sobrevida más larga (158 meses) presentaba un cistoadenocarcinoma de páncreas.

Un aspecto técnico discutible es si realizar una resección lateral o segmentaria completa de la VPVMS, algunos grupos prefieren la resección completa argumentando que es más oncológica ${ }^{24}$, sin embargo, no se ha demostrado diferencia entre las 2 técnicas ${ }^{11}$. Esta serie, como otras ${ }^{11}$, está compuesta en su mayoría de resecciones laterales, la cual consideramos una técnica de menor riesgo de complicaciones, tanto de sangrado como de trombosis a nivel de la sutura. Por supuesto la decisión de utilizar una u otra técnica depende también de la magnitud de la invasión de los vasos.

Una de las limitaciones de este estudio es su carácter retrospectivo de una cohorte relativamente pequeña de pacientes, en relación a grandes centros.

Esta serie confirma que la resección de VP-VMS asociada la PDD, es una terapia que si bien tiene una morbilidad elevada, permite una sobrevida comparable a pacientes con una PDD sin RV. Por este motivo, creemos que esta técnica está justificada para un grupo seleccionado de pacientes en buenas condiciones, sin patología grave asociada, de preferencia pacientes jóvenes, que no presentan diseminación a distancia, en los cuales el tumor invade focalmente la VP-VMS y su resección permite lograr una cirugía R0.

\section{Agradecimientos}

Especial agradecimiento a la Sra. Danitza Undurraga y Srta. Andrea Tello, secretarias de la oficina del cáncer por la obtención de datos y al Sr. Juan Ganci, estadístico del hospital por su colaboración en el seguimiento alejado de los pacientes.

\section{Referencias}

1. Winter J, Cameron J, Campbell K, Arnold M, Chang D, Coleman J, et al. 1423 pancreaticoduodenectomies for pancreatic cancer: A single-institution experience. J Gastrointest Surg 2006; 10: 1199-1210.

2. Tseng J, Raut C, Lee J, Pisters P, Vauthey J, Abdalla E, et al. Pancreaticoduodenectomy with vascular resection: margin status and survival duration. J Gastrointest Surg 2004; 8: 935-949.

3. Harrison L, Klimstra D, Brennan M. Isolated portal vein involvement in pancreatic adenocarcinoma. A contraindication for resection? Ann Surg 1996; 224: 342-349.

4. Leach S, Lee J, Charnsangavej C, Cleary K, Lowy A, Fenoglio C, et al. Survival following pancreaticoduodenectomy with resection of the superior mesentericportal vein confluence for adenocarcinoma of the pancreatic head. Br J Surg 1998; 85: 611-617.

5. Van Geenen R, Ten Kate F, de Wit L, Van Gulik T, Obertop H, Gouma D. Segmental resection and wedge excision of the portal or superior mesenteric vein during pancreatoduodenectomy. Surgery 2001; 129: 158-163.

6. Furhman G, Leach S, Stanley C, Cusack J, Charnsangavej C, Cleary K, et al. Rationale for en bloc vein resection in the treatment of pancreatic 
adenocarcinoma adherent to the superior mesentericportal vein confluence. Pancreatic Tumor Study Group. Ann Surg 1996; 223: 154-162.

7. De La Fuente H, Caracci M, Díaz A, Mege R, Raddatz A, Buchheister M, y cols. Pancreatoduodenectomía en el cáncer de la cabeza del páncreas. Rev Chil Cir 1994; 46: 470-476.

8. Waugh E. Pancreatogastroanastomosis en pancreatoduodenectomía. Una alternativa segura de anastomosis al muñón pancreático. Rev Chil Cir 2002; 54: 5964.

9. Horton K. Multidetector CT and three-dimensional imaging of the pancreas: state of the art. J Gastrointest Surg 2002; 6: 126-128.

10. Friess H, Ho C, Kleeff J, Buchler M. Pancreaticoduodenectomy, distal pancreatectomy, segmental pancreatectomy, total pancreatectomy, and transduodenal resection of the papilla of Vater. En: Surgery of the liver, biliary tract and pancreas. Philadelphia: Editorial Saunders Elsevier, 2007; 877-903.

11. Yekebas E, Bogoevski D, Cataldegirmen G, Kunze C, Marx A, Vashist Y, et al. En bloc vascular resection for locally advanced pancreatic malignancies infiltrating major blood vessels. Perioperative outcome and long term survival in 136 patients. Ann Surg 2008; 247: 300-309.

12. Bachellier P, Nakano H, Oussousltzoglou P, Weber J, Boudjema $\mathrm{K}$, Wolf $\mathrm{P}$, et al. Is pancreaticoduodenectomy with mesentericoportal venous resection safe and worthwhile? Am J Surg 2001; 182: 120-129.

13. Siriwardana H, Siriwardena A. Systematic review of outcome of synchronous portal-superior mesenteric vein resection during pancreatectomy for cancer. $\mathrm{Br} \mathrm{J}$ Surg 2006; 93, 662-673.

14. Birkmeyer J, Siewers A, Finlayson E, Stukel T, Lucas L, Batista I, et al. Hospital volume and surgical mortality in the united states. NEJM 2002; 346: 1128-1137.
15. Lieberman M, Kilburn H, Lindsey M, Brennan M. Relation of perioperative deaths to hospital volume among patients undergoing pancreatic resection for malignancy. Ann Surg 1995; 222: 638-645.

16. Allema J, Reinders M, van Gulik T, van Leeuwen D, de Wit L, Verbeek P. Portal vein resection in patients undergoing pancreatoduodenectomy for carcinoma of the pancreatic head. Br J Surg 1994; 81: 1642-1646.

17. Tashiro S, Uchino R, Hiraoka T, Tsuji T, Kawamoto $\mathrm{T}$, Saitoh N. Surgical indication and significance of portal vein resection in biliary and pancreatic cancer. Surgery 1991; 109: 481-487.

18. Poon R, Fan S, Lo C, Liu C, Lam C, Yuen, et al. Pancreaticoduodenectomy with en bloc portal vein resection for pancreatic carcinoma with suspected portal vein involvement. World J Surg 2004; 28: 602608.

19. Park J, Yoon Y, Kim Y, Ryu J, Yoon W, Lee S. Survival and prognostic factors of unresectable pancreatic cancer. J Clin Gastroenterol 2008; 42: 8691.

20. Butte JM, Norero E, Duarte I, Llanos O. Tumores quísticos del páncreas. Rev Chil Cir 2004; 56: 341 345.

21. Brugge W, Lauwers G, Sahani D, Fernández-del Castillo C, Warshaw A. Cystic neoplasms of the pancreas. NEJM 2004; 351: 1218-1226.

22. Bilimoria K, Talamonti M, Tomlinson J, Stewart A, Winchester D, Ko C. Prognostic score predicting survival after resection of pancreatic neuroendocrine tumors. Analysis of 3851 patients. Ann Surg 2008; 247: 490-500.

23. Roder J, Stein H, Siewert R. Carcinoma of the periampullary region: who benefits from portal vein resection?. Am J Surg 1996; 171: 170-174.

24. Ramacciato G, Mercantini P, Cucchetti A, Del Gaudio M. About pancreatic resection with portal vein resection. Ann Surg 2009; 249: 349-350. 